\author{
Andrzej NAPIERALSKI \\ Uniwersytet Łódzki \\ napieralski.a@op.pl
}

\title{
LES ATTAQUES VERBALES VISANT LES FOOTBALLEURS DANS LE DISCOURS DES INTERNAUTES
}

L'attaque verbale est une vaste notion qui détermine un phénomène regroupant des traits propres à différents types d'actes de paroles et particulièrement aux insultes. Le problème de définir ce qu'on entend par le mot insulte est de nature terminologique, ses équivalents - tels injure, invective, juron, blasphème, gros mot, incivilité, outrage et maintes autres ont tous un signifié particulier qui les distingue les uns des autres au niveau du sens. Le but de cet article n'est pas de s'interroger sur les différences sémantiques entre les termes énumérées, mais de présenter un corpus dans lequel l'attaque verbale prend une forme qui possède probablement des traits particuliers de toutes les appellations mentionnées ci-dessus. Le corpus que nous soumettons à l'analyse provient d'une sorte de forum de discussion sur Internet qui accompagne les articles de la version en ligne du fameux magazine sportif L'Equipe (www.lequipe.fr). Le caractère des énoncés présents sous les articles (et en général sur Internet) diffère beaucoup des formes écrites traditionnelles, aussi bien dans sa forme que dans son contenu. Internet dicte certains comportements qui sont considérés comme transgressant la norme du 'bon français', comme par exemple la liberté dans l'utilisation des signes diacritiques (certaines lettres sont dépourvues de ces marques même dans la correspondance officielle par mail pour éviter les problèmes de codage des logiciels) ou la simplification de l'orthographe des échanges désinvoltes sur la toile.

L'attaque verbale c'est au sens large une parole qui peut être blessante pour l'autre. Cependant, il n'est pas rare que ce soit la situation 
qui détermine le caractère offensant d'un mot ou d'une phrase qui ne l'est pas hors contexte. Il est donc possible de dire que l'acte de parole qui est l'insulte s'appuie sur un calcul du sens d'un énoncé où celui-ci n'appartient pas à l'axiologie lexicalisée usuelle. Chaque lieu de confrontation des idées sur la toile possède ses propres règles et ses codes langagiers et comportementaux qui se sont établies avec le temps et qui sont surtout le fruit des échanges des habitués qui s'y retrouvent. L'apparition d'une nouvelle personne sur un lieu d'échange d'idées sur Internet peut mettre celle-ci dans une situation un peu délicate à l'image de l'exemple qui suit : «La non-appartenance au groupe peut modifier la réception d'une insulte, créer un acte outrageant qui se voulait amical, ou au contraire amener l'allocutaire à ne pas se sentir émotivement concerné (un Français insulté par un Québécois avec des formes de sa variété pourra ne pas se sentir atteint, le percevant comme propre à un groupe extérieur)» (Lagorgette $2004: 7$ ). Les nouveaux lecteurs de certains forums peuvent être parfois confrontés à un problème de décryptage de la signification de certains signes qui sont souvent des abréviations et des sigles, voir même parfois des formes lexicales créées par les participants de ces forums. A l'image du fameux forum de trolls d'Internet polonais le 'Karachan', il se doit de constater que certains milieux sur le web sont fermés aux nouveaux adeptes (il faut avoir une recommandation d'un membre pour pouvoir postuler à l'accès à ce forum) et de plus on y retrouve des codes (p. ex. l'obligation pour tous d'utiliser le nom d'utilisateur 'anonymous' ou d'utiliser uniquement la forme masculine) et des pratiques langagières (argot des membres). Les exigences quant aux commentaires visant les articles sur le football dans lequipe.fr ne sont pas si sévères, toutefois il existe des conduites à respecter et des comportements à éviter.

Du fait que les moyens traditionnels d'insulter les footballeurs pendant les matchs de foot tels les 'gros mots' sont prohibés sur Internet, un système de contournement visant à déprécier le sportif a été établi. Les commentaires sur lequipe.fr sont dans la plupart des cas des réactions à un article : le plus souvent il s'agit de préciser une information, développer le sujet ou analyser le jeu d'une équipe. Les internautes puisent en général dans d'autres sources (comme leurs souvenirs ou d'autres sites Internet) pour faire des rappels historiques (sur les statistiques ou les matchs du passé) ou pour expliquer ce qui n'a pas été écrit dans l'article (par exemple les coulisses d'une affaire du type Af- 
faire Zahia ${ }^{1}$ ou Affaires des Quotas ${ }^{2}$. Cependant, il arrive que l'article touche un sujet sensible, ce qui fait tout de suite apparaître des commentaires virulents. Presque $90 \%$ des articles concernant le foot sur lequipe.fr sont libres de commentaires à caractère hostile envers les sportifs ou autres internautes, dans le reste figurent de vives contestations de plusieurs types.

Le but du présent travail étant d'analyser les attaques verbales dirigées vers les footballeurs, il ne sera pas question de l'échange de remarques et $\mathrm{d}^{\prime}$ insultes entre internautes. Internet donne la possibilité de s'exprimer librement et de transmettre ses pensées sur la toile sans censure (à part celle instaurée par les modérateurs de certains sites). Les gens abusent souvent de cette liberté en traitant Internet comme défouloir et lieu où ils pourront apaiser au moins un peu leur frustration. Le besoin d'attirer l'attention des autres est un autre facteur qui pousse les gens à 'poster' sur Internet en manifestant des animosités personnelles et surtout en cherchant à entretenir la rivalité. A part le caractère informatif, les commentaires jouent aussi un rôle ludique pour les lecteurs qui prennent plaisir à lire les remarques tranchantes et crues. Pour inciter les internautes à commenter les articles, il est essentiel que l'article possède un titre qui incite à discuter. Dans le monde du football il y a des idoles positives et négatives et les capacités sportives constituent rarement la raison de l'appartenance d'un footballeur à un groupe et non pas à un autre. Notons que les joueurs qui sont le plus souvent visés par les commentaires négatifs possèdent quelques traits communs. La haine et l'hostilité sont rarement désintéressées, pour 'mériter' l'attention des internautes un footballeur doit remplir quelques critères. Premièrement, il doit être populaire, jouer à un niveau international et être reconnu par la communauté des supporters du foot. Deuxièmement, le footballeur doit être actif dans les médias et sur les réseaux sociaux pour que ses opinions et ses propos soient connus. La dernière chose - et probablement la plus importante - c'est le caractère du footballeur et son 'unicité' qui font de lui un personnage qu'on ne peut pas aimer. Le milieu des supporters est plutôt unanime sur les footballeurs

1 Affaire dans laquelle 2 footballeurs de l'équipe de France de football ont été impliqués. Il s'agissait d'un cas de viol sur prostituée mineure.

2 Affaire dans laquelle l'entraîneur de l'équipe de France de football à l'époque Laurent Blanc a été accusé de racisme pour avoir contesté le système de formation des jeunes qui selon lui devrait favoriser les jeunes Français. 
qui méritent le respect et ceux qui sont jugés tout simplement 'cons' ou prétentieux. Une fois qu'on possède ce savoir il suffit de taper le nom du joueur dans le moteur de recherche du site et de choisir un article avec le nom du joueur pour pouvoir trouver des attaques verbales à l'égard de cette personne. Il est important de signaler qu'afin de respecter l'authenticité des commentaires analysés dans cette étude, toutes les productions des internautes sont données en italique à la forme exacte sous laquelle elles ont été trouvées. Les fautes orthographiques et grammaticales comme : le manque de lettres majuscules, les omissions de signes diacritiques ou le manque de l'accord entre le sujet et le verbe sont des fautes fréquentes qui constituent un trait identitaire pour les membres de la communauté internet. 10 articles de lequipe.fr ont été choisis pour cette analyse et le corpus de commentaires provient essentiellement des articles ci-dessous :

- Anelka ne jouera pas en Algérie (12/01/2015)

- Aidons Thauvin à avoir le déclic $(30 / 01 / 2015)$

- Thauvin-Payet, pas l'amour fou (08/02/2015)

- Ben Arfa, la piste chinoise (12/02/2015)

- Nasri : 'Ce sont des hypocrites' (13/02/2015)

- Giroud, joueur le plus sexy (18/02/2015)

- Quand Chris Waddle compare Samir Nasri à une Ford Fiesta $(26 / 02 / 2015)$

- Gignac, radiographie d'un buteur (06/03/2015)

- Ces joueurs plombés par la comparaison $(07 / 03 / 2015)$

- Franck Ribéry : 'Je me suis maîtrisé' (12/03/2015)

L'étude proposée distinguera trois catégories d'attaques verbales qui sont les plus présentes dans les commentaires des internautes, à savoir les figures, les insultes et les moqueries. En nous inspirant librement de la théorie des tropes de Dumarsais et des figures du discours discernées par Fontanier, nous avons décidé que les figures seraient divisées en celles de mots, de sens et de pensée. Certaines figures de mots sont des jeux sur les sonorités où les composants des syntagmes et des phrases donnent un effet de répétition des sons comme les assonances (Hatem Ben Thauvin? Je ne comprends plus rien...) ou les redoublements expressifs (Joueur sur-sur-sur côté). Les figures qui apparaissent dans les énoncés sont le plus souvent des constructions artificielles dont le but est d'amuser (l'utilisation de la rime) ou de manifester son point de vue d'une façon ostensible (la répétition du préfixe). Les commentaires des internautes ne 
manquent pas de noirceur ni de malveillance, le but de la plupart des gens est de s'amuser aux dépens du footballeur et de son geste hors du commun ou de sa déclaration dépassée.

La paranomase, qui «réunit dans la même phrase des mots dont le son est à peu-près le même, mais le sens tout-à-fait différent » (Fontanier 1977 : 347) est la figure qui est la plus présente dans les commentaires de ce type. Dans un des articles le footballeur Samir Nasri est comparé à une Ford Focus, ce qui pousse un internaute à jouer sur la sonorité en postant J'aurais plutot ${ }^{3}$ dit une Ford "faucu"..., locution homophonique au signifié 'faux cul' pour ce populaire modèle de la marque. L'homophonie est souvent utilisée dans les commentaires, dans l'exemple un "poids" chiche au service de la technique..., le lapsus qui confond les lexèmes 'pois' et 'poids' est volontaire pour faire remarquer qu'un joueur pourrait aller au régime. La paranomase joue souvent sur le nom de famille par allusion à une situation concrète. Notamment, la rumeur concernant le passage de Hatem Ben Harfa dans un club en Chine a été tout de suite utilisée par la communauté sur le forum. Ainsi, le patronyme du joueur, Ben, a vite été transformé en Nem (La Chine ça serait pas mal pour Nem Arfa!) ; dans Nem pas les perturbateurs en Chine... le syntagme verbal 'je n'aime' a été remplacé par 'nem' ; dans Je ris jaune le jeu s'appuie sur l'homophonie du verbe 'rire' à la première personne du présent de l'indicatif et du substantif 'riz'.

La contrepèterie constitue un autre jeu sur les sonorités qui est fréquemment exploité dans les commentaires. Cette figure est visible dans l'exemple Aidons Thauvic a avoir le déclin qui est un détournement du titre de l'article "Aidons Thauvin à avoir le déclic ». Parmi les figures de mots on peut observer des cas de créations avec le nom du joueur tels les fracto-compositions ${ }^{4}$. Dans l'exemple Tauvinthon le nom du joueur Thauvin est joint au mot marathon ce qui donne naissance à une forme lexicale qui signale la grande endurance du footballeur.

Le monde du sport voit toujours apparaître de nouveaux protagonistes qui grâce à leur jeunesse deviennent des héros du moment. La carrière d'un sportif est éphémère et peut durer une quinzaine d'années à condition que les blessures ne l'inquiètent pas, cependant les bons

\footnotetext{
3 Les exemples en italique sont des citations qui viennent directement d'Internet et qui seront présentées sans aucune modification de forme ni correction des fautes orthographiques ou grammaticales.

4 Voir Sablayrolles 2006 : 150.
} 
joueurs peuvent s'inscrire dans les esprits des supporters pour longtemps. Les nouveaux joueurs attirent toujours des comparaisons avec leurs homologues du passé et on voit sans cesse apparaître des nouveaux $\mathrm{Zi}$ dane ou Makélélé. Toutefois, si la comparaison dans le monde du football est d'habitude positive et concerne l'aspect sportif des joueurs, sur la toile ce n'est pas toujours le cas. Dans les commentaires, on voit souvent des comparaisons qui font allusion à des comportements négatifs de certaines personnalités du football dans le passé. Pour reconnaître le caractère de la comparaison, il faut avoir un minimum de connaissance dans le domaine en question, ainsi en lisant govou le futur Gascoigne, il faut savoir que mis à part les brillantes qualités footballistiques, ce joueur était surtout réputé pour ses nombreuses cuites qui sont le vrai objet de la comparaison de Sydney Govou dans cette locution. Le même modèle peut être aussi visible dans Thauvin, le déjà Dugarry qui renvoie à la rapide diminution des performances de Christophe Dugarry, qui après avoir gagné la Coupe du monde en 1998 a subi un déclin de la forme sportive. Mis à part les comparaisons à d'autres personnes, il en existe aussi celles qui touchent par exemple le physique de la personne. André-Pierre Gignac est un footballeur qui possède une silhouette un peu plus enveloppée que la moyenne des sportifs de haut niveau. Cela n'échappe pas à ses détracteurs qui utilisent souvent ce fait pour le déprécier comme dans Gignac, le futur Big Mac... où à la longue le nom du fameux sandwich McDonalds est même devenu son blase. Dans valbuena le futur adulte on retrouve aussi la comparaison liée au physique de la personne, car mesurant uniquement 1,67 cm Mathieu Valbuena est un footballeur plutôt petit. Les autres comparaisons qui ont été repérées dans le corpus sont liées à la culture de masse comme THAUVIN c'est un peu le magicien d'Oz. Une vraie ARNAQUE, voire à la tactique des clubs de foot comme gignacc est aussi le mec qui tire plus que le barça ne fait de passes.

La métaphore apparaît aussi souvent à côté de la comparaison dans les énoncés des internautes, comme dans Bravo chris! Nasri c come anelka, le ballon ils l ont sur les epaules où le ballon symbolise la tête qui n'est pas forcement remplie.

L'ironie, figure de pensée qui consiste à dire par raillerie l'inverse de ce que l'on pense est très présente dans les 'posts' des internautes. Son utilisation résulte essentiellement de l'aversion éprouvée pour certains joueurs et elle touche surtout les aspects mentaux de ceux-ci. Le présumé 
manque de courage de Florian Thauvin dans un cran au niveau du tir au pigeons? ou les problèmes avec le self-control de Franck Ribéry et Bravo attendre la trentaine pour apprendre à se maitriser! quelle maturité!!! sont des exemples fréquents de ce type de pratiques. Un point commun de la grande partie des joueurs qui sont critiqués sur la toile est constitué par leur supposé manque d'intelligence comme dans Avec la noix qui lui sert de cerveau, se maitriser était un exploit. C'est bien de le signaler. L'ironie, dans certains cas, peut être introduite grâce à un adjectif dont le but est d'exagérer le sens de l'énoncé. Dans les exemples Oui oui un grand joueur comme ben arfa mdr et Trop intégriste pour l'Algérie les adjectifs 'grand' et 'intégriste' sont utilisés comme déplacés dans le contexte des qualités des deux joueurs, du fait que l'un est jugé médiocre au niveau de ses capacités (grand) et l'autre trop fruste pour s'adapter à une autre réalité (intégriste).

Les insultes qui apparaissent sur la toile sont le plus souvent de nature délocutive, elles visent une personne qui est absente lors de l'apparition des énoncés. L'insulte que l'on trouve sur la toile «est fondée sur des jugements de valeur et est donc subjective. Elle est l'expression d'une pulsion. Sous l'emprise d'une émotion violente, le locuteur attribue au récepteur des propriétés nouvelles. Ce faisant, il lui construit une nouvelle identité, toujours négative. En effet, l'insulte dévalorise, disqualifie et blesse. Elle rabaisse également, voire nie l'autre parce qu'elle a pour socle les valeurs communes aux interlocuteurs» (Koffi-Lezou 2012 : 1). Les insultes tiennent leurs origines dans la vision subjective du monde de la personne qui les profère et qui est souvent influencée par la microsociété qu'elle côtoie. Le jugement personnel est très souvent à l'origine de l'apparition d'une insulte qui peut toucher le physique (tête a claques), le comportement (un mercenaire .., c'est une charrette, quelle supercherie ce type) ou encore le talent du footballeur (Nasri est un joueur en carton, Nasri surcôté ego démesuré, Un footeux). Il n'est pas rare non plus de trouver des jugements portant sur l'intelligence du joueur qui sont des propos fondés uniquement sur des convictions personnelles résultant de la sphère affective individuelle (Avec son qi d'huître il se maîtrise ... Pauvre garçon, quelle bêtise ce mec, De l'or dans les pied, Du vide dans la tête).

La plupart des internautes qui publient les commentaires sous les articles sont des supporters de football d'un club concret. L'animosité entre les clubs qui est venue d'Angleterre et qui se maintient dans le reste de 
l'Europe depuis presque un siècle 5 , le fameux phénomène des hooligans est le principal responsable du caractère que revêtent les relations entre des supporters aujourd'hui. La violence verbale qui existe dans les stades a gagné un nouveau terrain de confrontation - la toile. Les fans des clubs rivaux (comme ceux de l'OM et du $\mathrm{PSG}^{6}$ ) se confrontent souvent sur les forums et dans les commentaires à l'occasion des matchs de leurs clubs favoris. Dans l'exemple joueur surcoté comme toutes les marseillaises, le féminin est porteur d'une charge émotionnelle négative et d'une nuance dépréciative particulières.

La majorité des insultes adressées aux footballeurs sont des gestes qu'on qualifierait plutôt d'injurieux selon le critère de différence entre injure et insulte qui repose dans la véracité pour l'insulte et l'ironie pour l'injure. Comme le dit Maxime Chastaing : "Si donc vous pouvez utiliser le mot "parisien" [...] comme insulte en l'adressant à un Parisien, vous pouvez aussi l'utiliser comme injure en l'adressant à un individu que vous savez ne pas être parisien» (Chastaing $1980: 40$ ).

De nombreuses insultes ont leur source dans la popularité du footballeur : celui-ci voit apparaître une armée de 'rageux'7 à son égard par le simple fait qu'il gagne bien sa vie. Les exemples du corpus (Un petit con oui, et même un grand!, Déjà lui c'était pas un cadeau, ils nem pas les tetes de noud) témoignent d'un caractère non fondé des énoncés injurieux.

Une pratique souvent utilisée afin de déprécier quelqu'un est l'utilisation d'arguments rhétoriques, comme par exemple celui d'autorité, mais d'une façon ironique, souvent par antiphrase. Ainsi, l'évocation du fameux joueur de Barcelone, Andrés Iniesta, dans la phrase Iniesta aurait dû répondre après le match: "on mélange pas les torchons et les serviettes" mais il est bien trop humble et discret n'a d'autre rôle que d'injurier Samir Nasri, qui, selon l'auteur de l'énoncé n'était pas digne de se trouver sur la même pelouse que le grand joueur catalan. Tout comme dans le fameux énoncé de Coluche, selon qui «tout le monde se trouve intelligent puisqu'il se juge avec sa propre intelligence», où le syntagme sa propre intelligence évoque en fait, par antiphrase, la stupidité.

5 Selon Le Petit Robert le mot hooligan est entré en français en 1925 par le biais de la langue russe.

6 A savoir l'Olympique de Marseille et le Paris Saint-Germain.

7 Communauté d'internautes dont l'occupation principale est de critiquer et insulter gratuitement sur les forums. 
A côté de l'insulte dont le but est d'offenser et toucher le protagoniste, on a pu observer de nombreuses railleries à l'égard des footballeurs que l'on peut ranger dans la catégorie des moqueries. Dans le cadre de ce travail, les moqueries sont des propos à caractère souvent ironique et blessant dont le but est de tourner en ridicule quelqu'un sans se préoccuper de ses sentiments. Dans beaucoup de cas, les moqueries visent le physique d'une personne. Sous l'article consacré à «Giroud, joueur le plus sexy", on retrouve des exemples tels Heureusement pour lui, ribery ne joue pas en lère league ... ou il a gagné ce titre juste parce que Tevez a quitté le championnat anglais !!! qui sont des moqueries dont l'objet sont des joueurs au physique peu avantageux (aussi bien Franck Ribéry que Carlos Tevez possèdent des cicatrices respectivement sur le visage et sur le cou). Il arrive qu'un joueur soit comparé à un personnage de dessin animé, comme c'est le cas de Wayne Rooney qui a été déclaré Shrek ce qui explique l'exemple Rooney avec ces cheveux en plus ressemble de moins en moins à Shrek. La corpulence d'un footballeur peut lui procurer des commentaires comme C'est lequel sont burger prefere? comme c'est le cas d'André-Pierre Gignac. N'importe quoi peut faire l'objet d'une moquerie, il peut s'agir de l'âge avancé d'un joueur qui peut se faire railler par un propos ironique du type Parce qu'il y a des internationaux de moins de 27 ans qui vont en Algérie? Lolol ou du fait que le joueur change souvent de clubs Dommage ... un des rares pays où il n'avait pas joué ! Les footballeurs peuvent parfois se retrouver au chômage au moment où ils ne sont plus affiliés à un club, c'est alors l'occasion pour les 'fans' de proposer des solutions assez exotiques et absurdes comme Il lui reste Madagascar, va au pôle nord t'auras enfin le niveau, La piste martienne serait de loin la meilleure pour lui!, Et pourquoi pas les iles Fidji.......... La moquerie peut avoir comme origine un événement sur les réseaux sociaux, tel est le cas d'un 'tweet' de la femme de Samir Nasri qui a posté sur Twitter des insultes adressées à l'entraîneur de l'équipe de France qui n'a pas sélectionné son mari. Cette situation a vite été jugée par les internautes, qui depuis l'incident reviennent souvent à l'affaire en ridiculisant le footballeur et sa femme comme dans la moquerie On attend avec impatience le tweet de Madame Nasri... Remarquons à ce propos l'apparition d'un nouveau type de comportement sur Internet et les réseaux sociaux, appelé persiflage. Cette pratique concerne un nombre de plus en plus important de personnes appelées rageux ou trolls, selon l'occupation pratiquée. Leur activité sur Internet repose sur la critique et l'insulte de tout et de 
tout le monde sans idéologie, juste pour le plaisir et le défouloir. Les persiflages observés dans les commentaires peuvent être basés sur des connaissances culturelles. Ainsi, l'événement mondialement répandu de l'attaque des islamistes radicaux sur le siège du journal Charlie Hebdo et la fameuse phrase Je suis Charlie a donné naissance à des formes dérivées narquoises comme Je ne suis pas Nasri!!! Dans plusieurs cas, le persiflage a pour base une référence sportive, notamment les aptitudes des joueurs, leur talent Au moins il a ça parce que niveau talent le pauvre..., leur capacité à marquer des buts Un buteur. $M R D$, ou encore leur capacité à jouer en équipe il devrait faire un duo avec nasri : le collectif des incompris!

Une des caractéristiques des commentaires étudiés est que souvent ils s'enchaînent autour d'un seul motif à la manière d'un dénombrement où chacun rajoute son grain de sel. Sous l'article "Quand Chris Waddle compare Samir Nasri à une Ford Fiesta» on retrouve un répertoire de moqueries qui jouent toutes sur la comparaison du footballeur à la voiture.

- Une injure pour la Ford Fiesta...

- Aux dernières nouvelles, Ford songerait à porter plainte pour injure. Affaire a suivre...

- On prévoit une chute vertigineuse de l'action Ford à la Bourse...

- Ford a déposé une plainte pour insulte publique

- Des propos honteux et scandaleux de la part de Chris Waddle qui calomnie Ford.

- une Ford Fiesta, c'est fiable

- Une ford fiesta c'est encore trop grand pour lui!!!

- Nasri c'est plutôt une mobilette.

Les attaques verbales sont présentes sous différentes formes, notamment celles des mots, des syntagmes et même des phrases dont le but est de véhiculer un propos qui relève de l'insulte, de l'ironie ou de la moquerie. Les internautes qui commentent les articles sur la toile forment une communauté qui est arrogante et persuadée de l'infaillibilité de ses paroles. Cela les amène parfois à donner des 'conseils' aux footballeurs, de nature offensive en général, comme Mais qu'il se taise !!! va faire le coq avec ta poulette twiteuse ou prononcés d'une position de tuteur comme dans joueur d'une génération a qui il a manquait quelques gifles en étant gamin ça leur aurait appris le respect des autres!! Les 'conseils' sont le plus souvent des manifestations de mépris et d'indifférence envers les joueurs comme dans Qu'il aille jouer sur la lune, on s'en tape! ce qui laisse s'interroger 
sur les raisons de leur apparition. Il est certain que les insultes et les moqueries sont une conséquence d'une présence trop importante et souvent inutile du joueur dans les médias, qui lui amène la jalousie et la rage d'internautes. Le 'buzz' autour d'une personne célèbre est toujours considéré comme un manque de modestie de sa part et une manifestation de son insolence. Les défauts physiques et psychiques sont souvent l'objet des attaques verbales à l'égard des footballeurs, cela relève du caractère de certaines personnes complexées qui éprouvent le besoin d'avilir autrui. Bien évidemment, le comportement non professionnel de certains joueurs est aussi une raison de leur popularité négative sur la toile. Il est notamment considéré qu'un footballeur en tant qu'un personnage public devrait donner un bon exemple à de nombreux enfants pour qui il est une idole. Les animosités entre les clubs de différentes villes sont aussi à l'origine de la dépréciation de certains joueurs, toutefois, il arrive que parfois le manque de sympathie pour un joueur puisse découler d'une raison complètement aléatoire comme son apparence physique.

Les attaques verbales visant les footballeurs sont une pratique fréquente dans les commentaires sous les articles du site lequipe.fr. Les internautes sont en général unanimes quant aux noms de joueurs qu'il faut attaquer, car cela est strictement lié à un code comportemental qu'un footballeur professionnel devrait respecter aux yeux des supporters. Les attaques sont toujours le résultat d'un événement dans un passé plus ou moins proche, cependant une 'gaffe' commise par un joueur dans sa jeunesse pourra toujours ressortir dans le futur. Le but de poster des attaques visant les footballeurs sur la toile sont le plus souvent de nature à se défouler. Il existe néanmoins un certain rite de création des attaques qui est une sorte de jeu ayant comme cible de divertir la communauté des lecteurs des commentaires. Les commentaires réussis sont ceux qui relèvent de la créativité, de l'originalité et du bons sens, la présence d'un nombre de figures du discours témoigne d'une certaine 'recherche' stylistique des auteurs. Il est vrai que chaque personne peut écrire un commentaire sur le site du magazine l'Equipe, mais il n'est pas moins vrai que la connaissance du milieu et de la 'culture' des internautes habitués de ce site est essentielle pour pouvoir participer à l'échange d'attaques verbales et surtout à la compréhension de tous les commentaires. 


\section{RÉFÉRENCES}

Bonhomme M., 1999, «L'injure comme anticommunication », in : Violence et Langage, Actes du $19^{\text {e }}$ Colloque d'Albi LANGAGES et SIGNIFICATION, éds Robert Gauthier, Toulouse, 25-41.

Bustamante Arana R., 2006, "Agression et transgression : les tabous brisés du langage. Gros mots et vulgarité dans l'espagnol du Mexique», Revue d'Etudes Françaises, L'argot un universel du langage? $\mathrm{N}^{\circ}$ 11, Budapest, 11-27.

Chastaing M., Abdi H., 1980, «Psychologie des injures », Journal de psychologie 1, 31-62.

Drosik A., 2010, «W sieci Sieci. Jak Internet zmienia sferę publiczną », in : Władza mediózw W. Piątkowska-Stepaniak, A. Drosik (éd.), Wydawnictwo Uniwersytetu Opolskiego, Opole, 117-130.

Ernotte P., Rosier L., 2004, «L'ontotype : une sous-catégorie pertinente pour classer les insultes ? », Langue française 144, 24-48.

Fontanier P., 1977, Les figures du discours, Flammarion, Paris.

Koffi-Lezou A-D., 2012, «La violence verbale comme un exutoire. De la fonction sociale de l'insulte», Signes, Discours et Sociétés [en ligne], 8. La force des mots : valeurs et violence dans les interactions verbales, http://www.revuesignes.info/document.php?id=261

Lagorgette D., Larrivée P., 2004, «Introduction », Langue Française 144, 3-12.

Lehmann A., Martin-Berthet F., 1998, Introduction à la lexicologie, Nathan, Paris.

Rouayrenc C., 1996, Les gros mots, PUF, Paris.

Sablayrolles J-F., 2006, «La néologie aujourd'hui », in : A la recherche du mot : De la langue au discours, C. Gruaz (éd.), Lambert-Lucas, Limoges, 141-157.

\section{LES ATTAQUES VERBALES VISANT LES FOOTBALLEURS DANS LE DISCOURS DES INTERNAUTES}

\section{Résumé}

L'insulte c'est au sens large une parole qui peut être blessante pour l'autre. Cependant, il n'est pas rare que ce soit la situation qui détermine le caractère offensant d'un mot ou d'une phrase qui ne l'est pas sans contexte. Nous pouvons dire que l'acte de parole qui est l'insulte est ce calcul du sens d'un énoncé quand celui-ci n'appartient pas à l'axiologie lexicalisée usuelle. Dans notre contribution nous nous proposons de montrer comment s'opèrent les insultes dans les commentaires des internautes dans les articles sur le football. $\mathrm{Du}$ fait que les moyens traditionnels d'insulter les footballeurs pendant les matchs de foot tels les 'gros mots' sont prohibés sur Internet, un système de contournement visant à déprécier le sportif a été établi. Les mots et les locutions responsables pour ces outrages relèvent tantôt des procédés lexicogéniques 
(p. ex. le mot-valise thauvinthon du nom de joueur Florent Thauvin et du mot marathon) que des figures de style (p. ex. des comparaisons : un grand joueur comme ben arfa $m d r$, une charrette, un mercenaire ou une métathèse du titre de l'article "Aidons Thauvin à avoir le déclic » qui donne : Aidons Thauvic a avoir le déclin).

Mots-clés : football, lexicologie, analyse du discours, commentaires, Internet, sociolinguistique, insultes, langue des jeunes

\section{VERBAL ATTACKS ON FOTBALL PLAYERS IN THE DISCOURSE OF THE INTERNET USERS}

\section{Summary}

The insult is broadly a word that may be offensive. However, it is not rare that it is the situation that determines the offensive character of a word or phrase that is not offensive without context. We can say that the speech that is insulting is that calculation of the meaning of a statement when it is not up to the usual lexical axiology. We intend to show how insults are created in the comments of users in articles about football. Because the traditional methods of insulting footballers during football games like the "bad words" are prohibited on the Internet, a bypass system to depreciate the player was established. Words and phrases responsible for these outrages are created by lexicogenical processes (e.g. the portmanteau thauvinthon from the player's name Florian Thauvin and the word marathon) and figures of speech (e.g. ironical comparison to an other player: un grand joueur comme ben arfa mdr, comparison une charrette, un mercenaire or metathesis title of the article "Aidons Thauvin à avoir le déclic" which provides: Aidons Thauvic à avoir le déclin).

Key words: football, lexicology, discourse analysis, Internet, sociolinguistics, internet commentary, insults, youth speech 